\title{
Maximization of Energy-Efficiency under Convergence Constraint in Wireless Networked Control Systems
}

\author{
Mustafa Ozger Ozgur B. Akan \\ Next-generation and Wireless Communications Laboratory (NWCL) \\ Department of Electrical and Electronics Engineering \\ Koc University, Istanbul, Turkey \\ Email: \{mozger, akan\}@ku.edu.tr
}

\begin{abstract}
Wireless networked control system (WNCS) is a control system that a wireless network closes the control loop. WNCS estimator, i.e., Kalman filter, estimates the system state according to the observations of sensors. These observations which are from $N$ independent subnetworks are conveyed to the Kalman filter through vacant bands opportunistically with cognitive radio capability of the nodes. We characterize the successful packet delivery probability and study the maximization of energy-efficiency of overall system under the convergence constraint of the Kalman filter by defining an optimization problem. We also find a lower bound on maximum total coverage area. Furthermore, we perform numerical analysis to observe the effects of system parameters such as number of subnetworks, average $\mathrm{ON}$ probability of primary users, transmission ranges and densities of sensor nodes and primary users, and false alarm probability.
\end{abstract}

\section{INTRODUCTION}

Inefficient utilization of spectrum resources has triggered the use of dynamic spectrum access (DSA) techniques in wireless communications. The technology which enables DSA is cognitive radio (CR) [1]. A wireless network consisting of CR capable wireless nodes can opportunistically use the idle spectrum bands. However, a communicating pair of CRs must not cause any interference to active primary users (PUs). Spectrum sensing, spectrum decision and spectrum hand-off are the fundamental operations for opportunistic spectrum usage.

Apart from the inefficient utilization, some spectrum bands are very crowded due to excessive use. Wireless sensor networks (WSNs) suffer from the spectrum shortage. In order to overcome the spectrum scarcity challenge, cognitive radio sensor networks (CRSNs) are proposed [2]. They consist of wireless sensor nodes with CR capability. Most important aspect of CRSNs is that unnecessary energy consumption due to the spectrum scarcity can be decreased by the opportunistic spectrum usage. CRSNs can be used in diverse application areas.

Networked control system (NCS) is a traditional feedback control system closed via a communication network [3]. The communication network is utilized to exchange information between control system components such as sensor, controller, actuator, etc. [4], [5]. If the network has wireless communication capabilities with wireless control system components, the system becomes wireless networked control system (WNCS). It can be used in diverse application areas such as smart grid, automatic management and navigation systems [6].

The WNCS estimator estimates the system state according to the sensor observations. Hence, the convergence of the WNCS estimator becomes a fundamental issue during the design of a WNCS. For a WNCS, we consider that there are $N$ wireless subnetwork which are CRSNs such that the sensor measurements are conveyed to the WNCS estimator through idle channels opportunistically.

In our system, the WNCS estimator is a Kalman filter. In the classical approach, the Kalman filter has all the observation information to estimate the state of the system. On the other hand, the dynamic wireless channel conditions cause losses or delays for an observation to reach the Kalman filter. Hence, the observations can be intermittent. [7] studies the state covariance error for the general time-varying Kalman filter in which the observations are intermittent. However, the instability of the Kalman filter is not characterized. On the other hand, [8] models observation packet arrivals through wireless multihop sensor network as a random process and characterizes the statistical convergence properties of the Kalman filter. As an extension, [9] divides the state observation process into two parts such that there are two independent sensors providing observation packets to the Kalman filter and investigates the conditions for the convergence. Furthermore, in our prior work [10], we have generalized the division of the observation process in [9] by dividing the process into $N$ independent subnetworks and characterize the lower bound on maximum total coverage area. Furthermore, we investigate this lower bound in heterogeneous subnetworks which consist of PUs and CR nodes in [11].

In this paper, we also divide the process into $N$ parts. This corresponds to a WNCS with $N$ independent subnetworks, each of which consists of CR capable sensor nodes. Hence, the energy-efficiency is vital due to the resource-constrained nodes. Our aim is to convey the measurement packets by the energy limited sensor nodes to the Kalman filter in a dynamic radio environment under the convergence constraint of the Kalman filter. Hence, we maximize the energy-efficiency of the overall system. A new energy-efficiency function has been proposed for this purpose and an optimization problem is formulated to fulfill the requirements of the estimator 
convergence and the resource constraint. The contributions of the paper are the consideration of spectrum-aware communication in WNCS, the characterization of successful packet delivery probability and the maximization of energy-efficiency and finding lower bound on total network coverage area that guarantees the convergence of the Kalman filter.

The remainder of this paper is organized as follows. In Section II, convergence condition of Kalman filter with partial observations is presented. Section III presents our network model. In Section IV, the successful packet delivery probability is characterized. Section V presents our optimization problem and its solution. In Section VI, we provide the numerical analysis. Finally, the concluding remarks are given in Section VII.

\section{CONVERGEnce of Kalman Filter With PARTial OBSERVATIONS}

We divide the system into $N$ independent parts, hence, the system we consider can be represented as a general multiple-input multiple-output (MIMO) discrete time linear time-invariant system. The system equations can be written as follows

$$
\begin{gathered}
\mathbf{x}_{t+1}=A \mathbf{x}_{t}+\mathbf{w}_{t}, \\
{\left[\begin{array}{c}
\mathbf{y}_{1, t} \\
\vdots \\
\mathbf{y}_{N, t}
\end{array}\right]=\left[\begin{array}{c}
C_{1} \\
\vdots \\
C_{N}
\end{array}\right] \mathbf{x}_{t}+\left[\begin{array}{c}
\mathbf{v}_{1, t} \\
\vdots \\
\mathbf{v}_{N, t}
\end{array}\right]}
\end{gathered}
$$

where $\mathbf{x}_{t} \in \mathcal{R}^{n}$ is the system state vector, $\mathbf{y}_{i, t} \in \mathcal{R}^{m_{i}}$ is the sensor measurement vector, $\mathbf{w}_{t} \in \mathcal{R}^{n}$ is the system disturbance vector, $\mathbf{v}_{i, t} \in \mathcal{R}^{m_{i}}$ is the measurement noise vector, $A \in$ $\mathcal{R}^{n \times n}$ is the system matrix, and $C_{i} \in \mathcal{R}^{m_{i} \times n}$ is the output matrices for $i=1,2, \ldots, N$. The time index is indicated by the subscript $t$. Furthermore, the bold letters indicate the vectors. Due to the division of the system observation into $N$ parts, $\mathbf{y}_{t}=\left[\mathbf{y}_{1, t} ; \mathbf{y}_{2, t} ; \ldots ; \mathbf{y}_{N, t}\right], \mathbf{v}_{t}=\left[\mathbf{v}_{1, t} ; \mathbf{v}_{2, t} ; \ldots ; \mathbf{v}_{N, t}\right]$, and $C=\left[C_{1} ; C_{2} ; \ldots ; C_{N}\right]$. Both $\mathbf{w}_{t}$ and $\mathbf{v}_{t}$ are Gaussian random vectors with zero mean and their covariance matrices are $Q \geq 0$ and $R>0$, respectively. $R$ is a $N \times N$ matrix having elements as defined by $R_{i j}=\mathrm{E}\left[\mathbf{v}_{i, t} \mathbf{v}_{j, t}^{\prime}\right]$. If $(A, C)$ is observable, the Kalman filter converges without any loss in the sensor measurements.

All sensors measurement packets are received in a stochastic manner from different multi-hop cognitive wireless ad-hoc subnetworks by the Kalman filter in our system model as seen in Fig. 1. The stochastic reception of the packets is due to the wireless channel conditions. The sensors in these subnetworks encode their measurements as packets $\mathbf{y}_{1, t}, \mathbf{y}_{2, t}, \ldots, \mathbf{y}_{N, t}$. The arrival of each sensor measurement packet follows independent Bernoulli process, $\left\{\gamma_{i}\right\}_{t=0}^{t=\infty}$ with $\operatorname{Pr}\left\{\gamma_{i, t}=1\right\}=\lambda_{i}$ for $i=1,2, \ldots, N$ where $\gamma_{i, t}$ indicates the correct reception of the $i^{\text {th }}$ sensor measurement packet during a sample period. In each sample period $t$, the Kalman filter receives some of the output measurement packets due to the wireless channel conditions. The probability $\lambda_{i}$ depends on the channel characteristics.

It should be noted that for different time indexes $t$ and $t^{\prime}, \gamma_{i, t}$ and $\gamma_{j, t^{\prime}}$ for $i \neq j$ are independent. The loss of a packet is modeled as a reception of packet having an infinite noise variance. For the measurement noise vectors

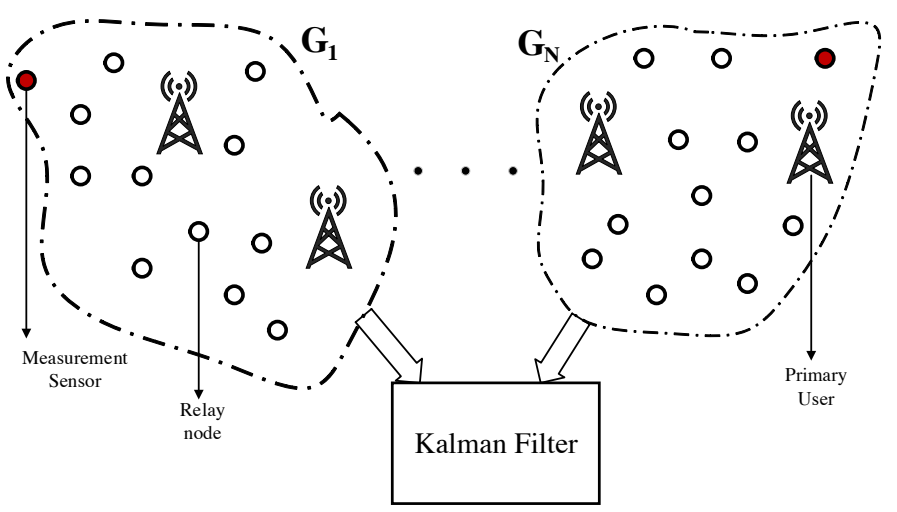

Fig. 1: A WNCS model consisting of $N$ subnetworks and a Kalman filter.

$\mathbf{v}_{i, t}$, we define the following conditional probability distribution function $f_{\mathbf{v} \mid \gamma}\left(\mathbf{v}_{i, t} \mid \gamma_{i, t}\right) \sim \mathcal{N}\left(0, R_{i i}\right)$ if $\gamma_{i, t}=1$ and $f_{\mathbf{v} \mid \gamma}\left(\mathbf{v}_{i, t} \mid \gamma_{i, t}\right) \sim \mathcal{N}\left(0, \sigma_{i}^{2} I\right)$ if $\gamma_{i, t}=0$. For random partial losses, we take $\sigma_{i}^{2} \rightarrow \infty$.

The important result of [8] has revealed that there exists a critical packet arrival probability such that if $\lambda>$ $\lambda^{c}$, the expected state estimation error covariance, $\mathrm{E}\left[P_{t+1 \mid t}\right]$ where $P_{t+1 \mid t}=E\left[\left(\mathbf{x}_{t+1}-\hat{\mathbf{x}}_{t+1 \mid t}\right)\left(\mathbf{x}_{t+1}-\hat{\mathbf{x}}_{t+1 \mid t}\right)^{\prime} \mid \mathbf{y}_{0}^{t}, \gamma_{0}^{t}\right]$, is bounded and $\mathrm{E}\left[P_{t+1 \mid t}\right]$ becomes infinite as $t \rightarrow \infty$ if $\lambda<\lambda^{c}$. In our prior work [10], we stated that based on the derivations and results given in [9], if $(A, Q)$ is controllable and $(A, C)$ is observable, for a fixed set of $\left(\lambda_{1}, \lambda_{2}, \ldots, \lambda_{i-1}, \lambda_{i+1}, \ldots \lambda_{N}\right)$, if $\lambda_{i} \geq \lambda_{i}^{c}$, the state estimation error covariance is bounded and the WNCS is stable. If the output matrices $C_{1}, C_{2}, \ldots, C_{N}$ are square and invertible $A$ has a single unstable eigenvalue, the upper and lower bounds for $\lim _{t \rightarrow \infty} \mathrm{E}\left[P_{t+1 \mid t}\right]$ coincide and the critical packet arrival probability of the measurement packet of the $i^{\text {th }}$ sensor becomes

$$
\lambda_{i}^{c}=\max \left\{0,1-\frac{1}{\alpha^{2} \prod_{\substack{j=1 \\ j \neq i}}^{N}\left(1-\lambda_{j}\right)}\right\}
$$

where $\alpha=\max _{i}\left|\sigma_{i}\right|$ and $\sigma_{i}$ is the $i^{\text {th }}$ eigenvalue of $A$ [9]. If all $\lambda_{i} \mathrm{~s}$ are greater than the critical values, the Kalman filter converges.

\section{Multi-Hop Wireless Subnetwork Model}

Sensors in each subnetwork transmit their observation packets to the Kalman filter over multiple hops in these subnetworks. Each subnetwork is distributed according to a two dimensional Poisson point process. These subnetworks are separated such that there is no connection between them. We assume that the nodes in the subnetworks have cognitive capability. Hence, nodes can sense the spectrum, change the operating parameters and adapt to the changes in the spectral environment. In fact, these subnetworks are CRSNs [2] such that output measurement packets are transmitted through available channel via multiple hops. Primary users (PUs), which are licensed users and have privilege to use the channel, and secondary users (SUs), which utilize the channel 
opportunistically, coexist in the subnetworks. $G_{i}$ indicates the $i^{\text {th }}$ subnetwork including the $i^{\text {th }}$ sensor node for our network system.

PUs are distributed according to a two dimensional Poisson point process with a density $\rho_{p}$. The transmission ranges of PUs, $r_{p}$, are the same. Furthermore, CRSN nodes are also distributed according to a two dimensional Poisson point process with a density $\rho_{s}$ and the transmission range of CRSN nodes is $r_{s}$. Fig. 1 shows our network model.

Although the spectrum availability changes in time for cognitive radio networks, there is a critical secondary node density $\rho_{s}^{*}$ which provides the connectivity of the network. In [13], the authors show that there exists a critical node density $\rho_{s}^{*}$ such that if the node density of the secondary network is larger than $\rho_{s}^{*}$, the secondary network percolates at all time, i.e., there exists always an infinite connected component in the secondary network under the time-varying spectrum availability. To guarantee the connectivity of the secondary network, we use the upper bound of the critical node density which is given by [13]

$$
\rho_{s}^{*}=\frac{5}{r_{s}^{2}} \ln \left[1-\sqrt{\left(1-(\sqrt{6} / 3)^{\Lambda}\right) e^{\left(\left|R_{e}\right|+\left|R_{e}^{\prime}\right|\right) \Pi_{1} \rho_{p}}}\right]^{-1}
$$

where $\Lambda=(4 L d+2 d+1) \times(4 L d+d)+(4 L d+d+1) \times(4 L d+$ $2 d)-1,\left|R_{e}\right|=\left(2+2\left\lceil r_{p} / d\right\rceil\right) \times\left(1+2\left\lceil r_{p} / d\right\rceil\right) \times d^{2},\left|R_{e}^{\prime}\right|=$ $\left(2+2\left\lceil r_{s} / d\right\rceil\right) \times\left(1+2\left\lceil r_{s} / d\right\rceil\right) \times d^{2}, L=\left\lceil\max \left\{r_{p}, r_{s}\right\} / d\right\rceil$, $d=r_{s} / \sqrt{5}$.

The state of PU can be represented as a semi-Markov ONOFF process, $S_{p}(t)$. OFF state is an spectrum opportunity for CRSN nodes since PU does not utilize the channel. On the other hand, ON state refrains CRSN nodes from communicating. Furthermore, $\Pi_{1}$ in (3) is defined as $\Pi_{1}=\operatorname{Pr}\left\{S_{p}(t)=1\right\}$. That is, $\Pi_{1}$ is the average ON probability.

To investigate the lower bound on the maximum coverage area of a WNCS, we assume that the network density is $\rho_{s}^{*}$ such that the nodes in subnetworks percolates all the time.

The transmission energy at one hop is $E_{t x}\left(N_{p}, r_{s}\right)=$ $E_{\text {elec }} \times N_{p}+\epsilon_{\text {amp }} \times N_{p} \times r_{s}^{2}$ where $E_{\text {elec }}=50 \mathrm{~nJ} / \mathrm{bit}$, $\epsilon_{a m p}=100 \mathrm{pJ} / \mathrm{bit} / \mathrm{m}^{2}, N_{p}$ is measurement packet size [14]. The reception energy is defined as $E_{r x}\left(N_{p}\right)=E_{e l e c} N_{p}$. During the transmission and reception of the measurement packet at one hop, energy is consumed at both transmitter and receiver side which can be written as

$$
E_{\text {hop }}^{\text {total }}=E_{t x}\left(N_{p}, r_{s}\right)+E_{r x}\left(N_{p}\right) .
$$

\section{Successful Packet Delivery Probability}

The delivery of a packet between two CR capable nodes depends on link establishment between these two nodes. The first condition of the link establishment is that the two nodes have to be located within the transmission ranges of each other. The second condition is to have a common channel between these two nodes. Furthermore, the common channel must not be sensed as occupied. This wastes the spectrum opportunity and fails the link establishment. Fig. 2 shows the two CR nodes which are within the transmission range of each other. The inner circles with solid lines show the maximum transmission ranges of the nodes which are $r_{s}$. There must be no active

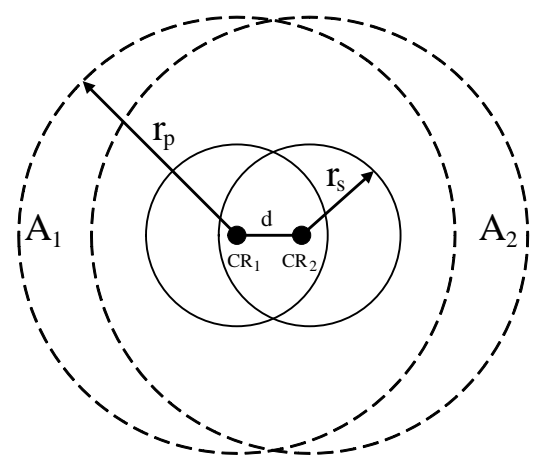

Fig. 2: Coverage of CR nodes and their guard ring for the link establishment.

PU around these nodes to establish a communication link between them. Hence, we determine a guard ring to facilitate spectrum-aware communication. This guard ring is determined by the union of circles whose radius is $r_{p}$. This provides an interference-free environment for the communicating nodes.

We denote the guard ring region covered by $C R_{1}$ and $C R_{2}$ with $A_{1}$ and $A_{2}$, respectively. The areas of these regions are $A_{1}=A_{2}=\pi r_{p}^{2}$. We previously stated that there must not be any active PU in the union of these regions to establish a link between $C R_{1}$ and $C R_{2}$. Hence, the link establishment probability from $C R_{1}$ to $C R_{2}$ can be found as

$$
\begin{aligned}
p_{l}(d)= & \operatorname{Pr}\left\{N o P U \text { in } A_{1} \cup A_{2}\right\} P_{F A}^{\prime} \\
& +\operatorname{Pr}\left\{P U \text { in } A_{1} \cup A_{2}\right\} \Pi_{1}^{k} P_{F A}^{\prime}
\end{aligned}
$$

where $P_{F A}^{\prime}$ is no false alarm probability and $\Pi_{1}$ is the ON probability of PU and $k$ is the mean number of PUs in $A_{1} \cup A_{2}$, $k=\rho_{p} \times\left(A_{1} \cup A_{2}\right)$, and $\rho_{p}$ is the PU node density.

The area of the region $A_{1} \cup A_{2}=A(d)$ can be found as

$$
A_{1} \cup A_{2}=2 \pi r_{p}^{2}-2 r_{p}^{2} \arccos \left(\frac{d}{2 r_{p}}\right)+\frac{1}{2} d \sqrt{\left(4 r_{p}^{2}-d^{2}\right)}
$$

where $d$ is the distance between the two nodes. $p_{l}(d)$ can be written as

$$
\begin{aligned}
p_{l}(d) & =e^{-\rho_{p} A(d)}\left(1-P_{F A}\right) \\
& +\left(1-e^{-\rho_{p} A(d)}\right) \Pi_{1}^{k}\left(1-P_{F A}\right) .
\end{aligned}
$$

We set $d$ to its maximum value $r_{s}$ in our calculations to calculate the worst case for the link establishment probability, $p_{l}$. This probability is lower bounded by the probability when $d=r_{s}$. Then, the constant link establishment probability is

$$
\begin{aligned}
p_{l}\left(r_{s}\right) & =e^{-\rho_{p} A\left(r_{s}\right)}\left(1-P_{F A}\right) \\
& +\left(1-e^{-\rho_{p} A\left(r_{s}\right)}\right) \Pi_{1}^{k}\left(1-P_{F A}\right) .
\end{aligned}
$$

As a routing protocol we employ the greedy forwarding approach [12]. In this routing approach, the node sends its packet to its neighbor node that is closest to the destination. We define a random variable $H$ which indicates that there exists at least one neighbor node in the forwarding region and it can be expressed as

$$
H= \begin{cases}1, & \text { if } \exists \text { a node in the forwarding region } \\ 0, & \text { otherwise. }\end{cases}
$$




$$
\begin{gathered}
\underset{\lambda_{1}, \ldots, \lambda_{N}}{\operatorname{maximize}} U\left(\lambda_{1}, \ldots, \lambda_{N}\right)=\lambda_{1} E_{1}^{\text {total }}+\lambda_{2} E_{2}^{\text {total }}+\cdots+\lambda_{N} E_{N}^{\text {total }} \\
\text { subject to } \lambda_{i} \geq \max \left\{0,1-\frac{1}{\alpha^{2} \prod_{\substack{j=1 \\
j \neq i}}^{N}\left(1-\lambda_{j}\right)} \text { for } i=1,2, \ldots, N .\right.
\end{gathered}
$$

In our calculations, we assume the forwarding region as the closer half of the disc to the destination specified by the transmission range of the sender node. There must be at least one node to successfully forward the packet to the destination. The successful packet delivery probability can written as

$$
p_{s}=p_{s \mid H=1} \operatorname{Pr}\{H=1\}+p_{s \mid H=0} \operatorname{Pr}\{H=0\}
$$

where $\operatorname{Pr}\{H=1\}=1-e^{-\rho_{s} \pi r_{s}^{2} / 2}$ and $\operatorname{Pr}\{H=0\}=$ $e^{-\rho_{s} \pi r_{s}^{2} / 2}$. The successful packet transmission probability when there is no neighbor in the forwarding region, $p_{s \mid H=0}$, is zero since there is no neighbor to forward the packet. The successful packet delivery probability when there is at least one neighbor in the forwarding region, $p_{s \mid H=1}$, is the link establishment probability, $p_{l}\left(r_{s}\right)$. Hence, the successful packet delivery probability becomes as

$$
\begin{aligned}
p_{s} & =p_{s \mid H=1} \operatorname{Pr}\{H=1\} \\
& =p_{l}\left(r_{s}\right)\left(1-e^{-\rho_{s} \pi r_{s}^{2} / 2}\right) .
\end{aligned}
$$

\section{MAXIMUM ENERGY-EFFICIENCY UNDER CONVERGENCE CONSTRAINTS}

Each subnetwork has energy-constrained sensor nodes, hence, the energy to convey the measurement packets to the Kalman filter must be minimized. On the other hand, there is a convergence constraint of the Kalman filter, which states that the probability of measurement packet reception correctly must be above a certain threshold for each subnetwork.

Coverage can be increased if we increase the number of nodes. However, it will increase the number of hops to reach the Kalman filter which decreases the probability of sensor measurement packet reception by the Kalman filter and increases total energy consumption to convey the measurement packets. Hence, increasing the number of hops may deteriorate the convergence of the Kalman filter and the energy consumption. Hence, we define hop diameter as a parameter which defines the maximum number of hops between any point in the network. Due to the convergence constraint, hop diameter becomes a critical parameter for the stability of the system. We denote the hop diameter of the subnetwork $G_{i}$ as $d_{i}$.

We define the critical packet arrival probability for each sensor measurement denoted by $\lambda_{i}^{c}$. It directly affects the hop diameter of a subnetwork and the critical hop diameter that guarantees the convergence of the Kalman filter is found as $d_{i}^{c}=\left\lfloor\ln \left(\lambda_{i}^{c}\right) / \ln \left(p_{s}\right)\right\rfloor+1$. In this case, if $d_{i}<d_{i}^{c}$ for any subnetwork $G_{i}$, WNCS is stable and the Kalman filter converges since it is guaranteed that the packet arrival probability is greater than the critical value. Otherwise, the system stability and the convergence of the Kalman filter are not guaranteed.
The total energy consumption in a subnetwork $G_{i}$ can be expressed as $E_{i}^{\text {total }}=d_{i} \times E_{\text {hop }}^{\text {total }}$. For a given set of packet arrival probabilities of the subnetworks except for the $i^{\text {th }}$ subnetwork, $\left(\lambda_{1}, \lambda_{2}, \ldots, \lambda_{i-1}, \lambda_{i+1}, \ldots \lambda_{N}\right)$, the convergence criterion states that as $\lambda_{i}^{c} \rightarrow 0$, then $d_{i}^{c} \rightarrow \infty$. While considering the convergence criterion, the critical hop diameter for $G_{i}$ becomes infinity and the energy consumed in this subnetwork to convey the measurement packet to the Kalman filter becomes infinity. This energy allocation is not efficient for a subnetwork that consists of energy-constrained nodes. Hence, the energy-efficiency of overall system must be considered.

The energy-efficiency of the overall network can be calculated by considering weights for subnetworks. The subnetworks having smaller critical packet arrival probability should have less weight. When $\lambda_{i}^{c} \rightarrow 0, d_{i}^{c} \rightarrow \infty$ and $\lambda_{i} E_{i}^{\text {total }} \rightarrow 0$. Hence, we can use $\lambda_{i} E_{i}^{\text {total }}$ as energy efficiency of the $i^{\text {th }}$ subnetwork. We therefore define the total energy-efficiency function of the WNCS as

$$
U\left(\lambda_{1}, \ldots, \lambda_{N}\right)=\lambda_{1} E_{1}^{\text {total }}+\lambda_{2} E_{2}^{\text {total }}+\cdots+\lambda_{N} E_{N}^{\text {total }} .
$$

We find the set $\left(\lambda_{1}, \lambda_{2}, \ldots, \lambda_{N}\right)$ which maximizes the energyefficiency in the system to convey the measurement packets to the Kalman filter under the convergence constraint of the Kalman filter which is defined in (2). The optimization problem is formulated as in (13).

We perform numerical analysis to obtain a solution to the optimization problem. The constraints on the packet arrival probability change the total energy consumption of the system. In our observations, the critical arrival probability should be the same for each subnetwork to maximize the energy-efficiency. Without considering the constraints on $p_{s}$ and $\alpha$, the maximum energy-efficiency is achieved by $e^{-\ln \left(p_{s}\right)-1}$ for small values of $\lambda_{i}$. For high values of $\lambda_{i}$, the maximum energy-efficiency can be approximated by $1-\alpha^{-2 / N}$. Hence, the optimum solution can be approximated as

$$
\lambda_{i}^{\mathrm{opt}} \approx \max \left\{e^{-\ln \left(p_{s}\right)-1}, 1-\alpha^{-2 / N}\right\}
$$

for $i=1,2, \ldots, N$, which guarantees the convergence of the Kalman filter and achieves the maximum energy-efficiency of the system.

The energy-efficiency maximization imposes a bound on the number of nodes in each subnetworks. If the number of nodes in a subnetwork is equal to the hop diameter, we guarantee the convergence of the Kalman filter since the packet arrival probability is smaller than the critical value. Hence, we can find the lower bound on the maximum number of nodes in the system. This means the lower bound on the maximum coverage with the maximum energy-efficiency under the convergence constraint of the Kalman filter. The lower bound on the maximum number of node is denoted by 


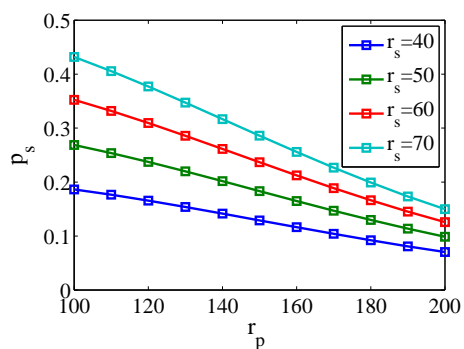

(a)

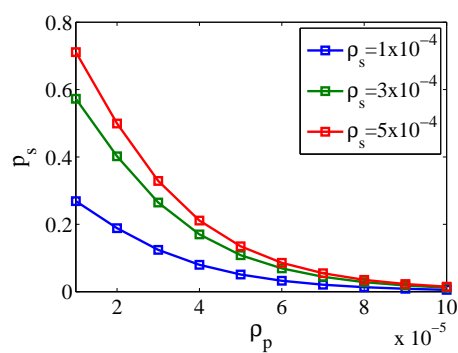

(b)

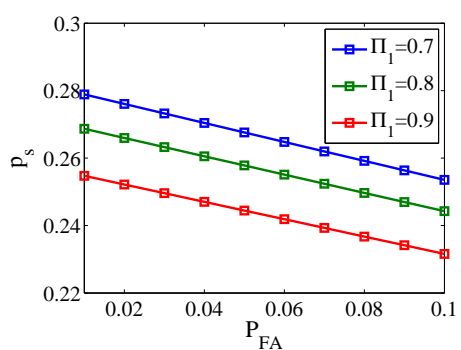

(c)

Fig. 3: Successful packet delivery probability, $p_{s}$, vs. a) $P_{F A}$ for different $\Pi_{1}$, b) $\rho_{p}$ for different $\rho_{s}$, c) $r_{p}$ for different $r_{s}$.

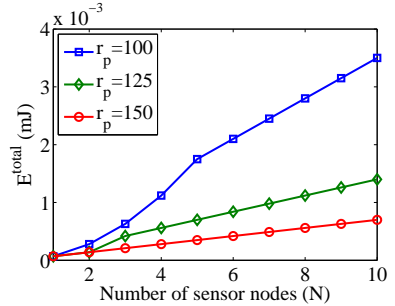

(a)

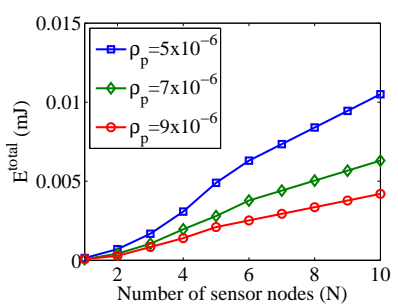

(b)

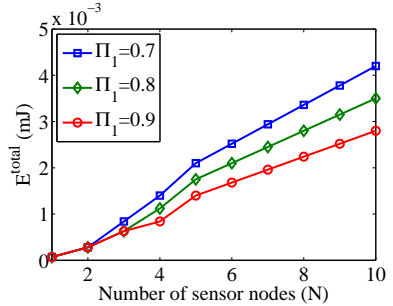

(c)

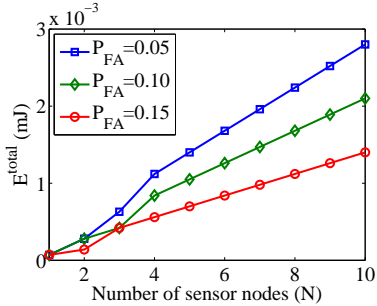

(d)

Fig. 4: Total energy consumption, $E^{\text {total }}$, vs. $N$ for different a) $r_{p}$, b) $\rho_{p}$, c) $\Pi_{1}$, d) $P_{F A}$.

$L_{i}=d_{i}^{\text {opt }}$ for $i=1,2, \ldots, N$. In Section III, we stated that for $\rho_{s}^{*}$, the network percolates at all time. Hence, we can find the coverage area of $G_{i}$ as $S_{i}=L_{i} / \rho_{s}^{*}$. The total coverage area of the system can be given by $S^{\text {total }}=\sum_{i=1}^{i=N} S_{i}=N \times S_{i}$ due to the same critical packet arrival probability value for each subnetwork.

\section{NUMERICAL ANALYSIS}

We perform numerical analysis of the successful packet delivery probability, $p_{s}$, the total energy consumption, $E^{\text {total }}=$ $\sum_{i=1}^{i=N} E_{i}^{\text {total }}$, and the lower bound on the maximum total coverage area, $S^{\text {total }}$ of the subnetworks with respect to several parameters of the system and multi-hop cognitive wireless subnetworks. The numerical values of system parameters are set as $\rho_{s}=10^{-4}$ nodes $/ \mathrm{m}^{2}, \rho_{p}=10^{-5}$ nodes $/ \mathrm{m}^{2}, \Pi_{1}=0.8$, $P_{F A}=0.01, r_{p}=100 \mathrm{~m}$, and $r_{s}=50 \mathrm{~m}$ unless otherwise specified. Furthermore, the output matrices $C_{1}, C_{2}, \ldots, C_{N}$ are assumed to be square and invertible so that the upper and lower bounds of the critical arrival probability coincide [9].

\section{A. Successful Packet Delivery Probability}

The successful packet delivery, $p_{s}$, depends on $r_{s}, r_{p}, \rho_{s}$, $\rho_{p}, \Pi_{1}$ and $P_{F A}$ as shown in (11). In Fig. 3(a), we investigate the effects of $r_{p}$ and $r_{s}$. As $r_{s}$ increases, the forwarding region expands and $P\{H=1\}$ increases. Hence, $p_{s}$ increases with an increase in $r_{s}$. Furthermore, as $r_{p}$ increases, $A_{1} \cup A_{2}$ expands. Hence, it would be less likely to find a neighbor to forward the packet towards the Kalman filter. As $r_{p}$ increases $p_{s}$ decreases.

As seen in Fig. 3(b), higher $\rho_{s}$ means higher number of neighbor to choose to forward the measurement packet. An increase in $\rho_{s}$ increases $p_{s}$. Also, $\rho_{p}$ has negative effect since it increases the number of possible PUs in $A_{1} \cup A_{2}$ region. Hence, an increase in $\rho_{p}$ decreases $p_{s}$.

As shown in Fig. 3(c), the average ON probability of PUs, $\Pi_{1}$, determines the idleness of the channel in $A_{1} \cup A_{2}$, and hence an increase in $\Pi_{1}$ decreases $p_{s}$. In terms of $P_{F A}$, an increase in $P_{F A}$ increases the chance of spectrum opportunity losses. Hence, an increase in $P_{F A}$ decreases $p_{s}$ as seen in Fig. 3(c).

\section{B. Total energy consumption}

We investigate the total energy consumption, $E^{\text {total }}$, with respect to the number of subnetworks, $N$, for different $r_{p}, \rho_{p}$, $\Pi_{1}$ and $P_{F A}$.We select the measurement packet size, $N_{p}$, as 200 bits. The energy consumption at each hop, $E_{\text {hop }}^{\text {total }}$, is equal to $0.06 \mathrm{~mJ}$.

In Fig. 4(a), $E^{\text {total }}$ with respect to the number of sensor nodes $N$ is investigated for different $r_{p}$ values. $E^{\text {total }}$ depends on $\lambda_{i}^{\text {opt }}=\max \left\{e^{-\ln \left(p_{s}\right)-1}, 1-\alpha^{-2 / N}\right\}$. For small $N$ values, $\lambda_{i}^{\text {opt }}=1-\alpha^{-2 / N}$ and we observe non-linear increase in $E^{\text {total }}$. For greater values of $N, \lambda_{i}^{\text {opt }}=e^{-\ln \left(p_{s}\right)-1}$ and we observe a linear increase as shown Fig. 4(a) due to $E^{\text {total }}=$ $N E_{i}^{\text {total }}$. Furthermore, $\rho_{p}$ affects the packet successful packet delivery probability, $p_{s}$. Hence, $\rho_{p}$ changes the value of the transition from the quadratic region to the linear region due to $\lambda_{i}^{\mathrm{opt}}$ expression. As we observed in Fig. 4(b), an increase in $\rho_{p}$ decreases $p_{s}$, and hence it will decrease this boundary as seen in Fig. 4(b).

The successful packet delivery probability, $p_{s}$, decreases with increasing $\Pi_{1}$. Hence, it decreases the hop diameter, 


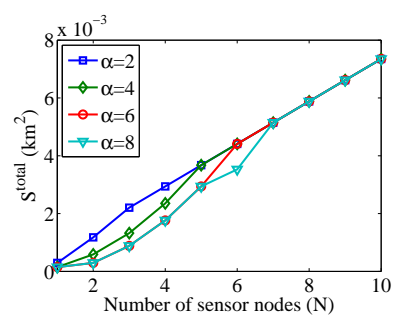

(a)

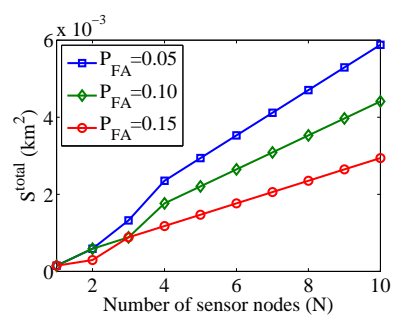

(b)

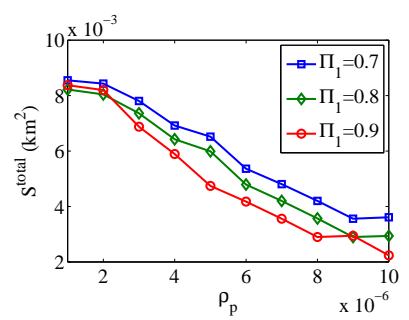

(c)

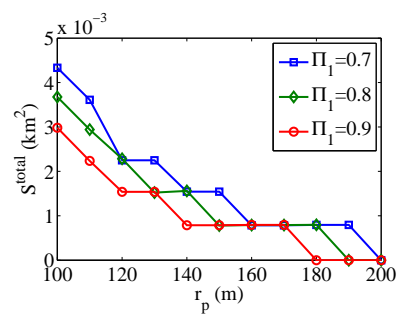

(d)

Fig. 5: Total coverage area, $S^{\text {total }}$, vs. a) $N$ for different $\alpha$, b) $N$ for different $P_{F A}$, c) $\rho_{p}$ for different $\Pi_{1}$, d) $r_{p}$ for different $\Pi_{1}$.

$d_{i}^{\mathrm{opt}}=\left\lfloor\frac{\ln \left(\lambda_{i}^{\mathrm{opt}}\right)}{\ln \left(p_{s}\right)}\right\rfloor+1$. This results in a decrease in $E^{\text {total }}$ as seen in Fig. 4(c). Furthermore, an increase in $P_{F A}$ decreases $p_{s}$. Since this effect decreases $d_{i}^{\text {opt }}, E^{\text {total }}$ decreases as seen in $4(d)$.

\section{Maximum Total Coverage Area}

In this section of the numerical analysis, we present the effect of $\alpha, P_{F A}, \Pi_{1}, \rho_{p}$ and $r_{p}$ on the lower bound of the maximum total coverage area of the subnetworks, $S^{\text {total }}$. We stated in Section $\mathrm{V}$ that the lower bound on the maximum number of nodes in subnetwork $G_{i}$ is $d_{i}^{\text {opt }}$. In Fig. 5(a), the variation in $S^{\text {total }}$ with respect to $N$ is investigated for different $\alpha$ values. The values of $\alpha$ and $N$ affect the $\lambda_{i}^{\text {opt }}$, and hence the lower bound on the maximum number of nodes in $G_{i}$. The successful packet delivery probability does not depend on both $N$ and $\alpha$. According to $\lambda_{i}^{\text {opt }}=\max \left\{e^{-\ln \left(p_{s}\right)-1}, 1-\alpha^{-2 / N}\right\}$ expression, for smaller values of $N, \lambda_{i}^{\text {opt }}=1-\alpha^{-2 / N} . S^{\text {total }}$ is non-linear below certain $N$. In the other case, $\lambda_{i}^{\text {opt }}=e^{-\ln \left(p_{s}\right)-1}$. Since $S^{\text {total }}=N S_{i}^{\text {total }}$, after a certain $N$ value, $S^{\text {total }}$ increases with only $N$. Furthermore, the probability of false alarm decreases successful packet delivery probability, $p_{s}$. Hence, an increase in $P_{F A}$ decreases $d_{i}^{\text {opt }}$. This decreases $S^{\text {total }}$ which can be seen in Fig. 5(b).

The PU density, $\rho_{p}$, has a negative impact on $S^{\text {total }}$ since an increase in $\rho_{p}$ decreases $p_{s}$ and it results in smaller $d_{i}^{\text {opt }}$. This effect can be seen in Fig. 5(c). Hence, an increase in $\Pi_{1}$ decreases $S^{\text {total }}$. Also, $\Pi_{1}$ decreases $S^{\text {total }}$ due to the decrease in $p_{s}$. The successful packet delivery probability states that an increase in $r_{p}$ causes a decrease in $p_{s}$. Hence, it decreases the total coverage area, $S^{\text {total }}$, as can be seen in Fig. 5(d).

\section{CONCLUSION}

In this paper, we maximize the energy-efficiency of a WNCS consisting of $N$ independent subnetworks having CR capable sensor nodes. We define an energy-efficiency function and formulate an optimization problem. We solve it under the convergence constraint of the Kalman filter. We characterized successful packet delivery probability and find a lower bound on the maximum coverage of the overall system which guarantees the convergence of the Kalman filter. We investigate the effects of system parameters and spectrumaware communication parameters on the successful packet delivery probability, the energy consumption and the lower bound on the maximum total coverage area of the system.

\section{ACKNOWLEDGMENT}

This work was supported by The Scientific and Technological Research Council of Turkey (TUBITAK) under grant \#110E249.

\section{REFERENCES}

[1] I. F. Akyildiz, W. Y. Lee, M. C. Vuran, and S. Mohanty, "NeXt generation / dynamic spectrum access / cognitive radio wireless networks: A survey," Computer Networks (Elsevier), Sept. 2006.

[2] O. B. Akan, O. Karli, and O. Ergul, "Cognitive radio sensor networks," IEEE Network, vol. 23, no. 4, pp. 34-40, Jul. 2009.

[3] R. A. Gupta and M.-Y. Chow, "Networked control system: Overview and research trends," IEEE Trans. Ind. Electron., vol. 57, no. 7, pp. 2527-2535, Jul. 2010.

[4] Y. Halevi and A. Ray, "Integrated communication and control systems: Part I-Analysis," J. Dynamic Syst., Measure. Contr., vol. 110, pp. 367373, Dec. 1988.

[5] M.S. Branicky, S.M. Phillips, and W. Zhang, "Stability of networked control systems: Explicit analysis of delay," in Proc. Amer. Control Conf., Chicago, IL, June 2000, pp. 2352-2357.

[6] A. Bemporad, M. Johansson, and M. Heemels, Networked Control Systems. Berlin, Germany, Springer, 2010.

[7] T. Fortmann, Y. Bar-Shalom, M. Scheffe, and S. Gelfand, "Detection thresholds for tracking in clutter - a connection between estimation and signal processing," IEEE Trans. Automat. Contr., vol. AC-30, pp. 221228, Mar. 1985.

[8] B. Sinopoli, L. Schenato, M. Franceschetti, K. Poola, M. I. Jordan, and S. S. Sastry, "Kalman filtering with intermittent observations," IEEE Trans. Autom. Control, vol. 49, no. 9, pp. 1453-1464, Sep. 2004.

[9] X. Liu and A. Goldsmith, "Kalman filtering with partial observation losses," in Proc. IEEE Conf. Decision and Control, Bahamas, vol. 4, pp. 4180-4186, Dec. 2004.

[10] D. Kilinc, M. Ozger, and O. B. Akan, "On the Maximum Coverage Area of Wireless Networked Control Systems under Stability and CostEfficiency Constraints," in Proc. IEEE GLOBECOM 2013, Atlanta, GA, USA, December 2013, pp. 274-279.

[11] D. Kilinc, M. Ozger, and O. B. Akan, "On the Maximum Coverage Area of Wireless Networked Control Systems with Maximum Cost-Efficiency under Convergence Constraint," in IEEE Transactions on Automatic Control, 2014.

[12] B. Karp and H. T. Kung, "GPSR: Greedy Perimeter Stateless Routing for Wireless Networks," in Proc. of ACM Mobicom, pp. 243-254, 2000.

[13] P. Wang, I. F. Akyildiz, and A. M. Al-Dhelaan, "Dynamic connectivity of cognitive radio ad-hoc networks with time-varying spectral activity," in Proc. of IEEE GLOBECOM 2010.

[14] W. Heinzelman, A. Chandrakasan, and H. Balakrishnan, "Energy Efficient Communication Protocol for Wireless Microsensor Networks," in Proc. of HICSS 2000, 2000. 